\title{
Long-lived Charginos in the Focus-point Region of the MSSM Parameter Space
}

\author{
A. V. Gladyshev ${ }^{1,2}$, D. I. Kazakov ${ }^{1,2}$ and M. G. Paucar ${ }^{1}$ \\ ${ }^{1}$ Bogoliubov Laboratory of Theoretical Physics, \\ Joint Institute for Nuclear Research, \\ 141980, 6 Joliot-Curie, Dubna, Moscow Region, Russian Federation \\ ${ }^{2}$ Institute for Theoretical and Experimental Physics, \\ 117218, 25 Bolshaya Cheremushkinskaya, Moscow, Russian Federation
}

\begin{abstract}
We analyse the possibility to get light long-lived charginos within the framework of the MSSM with gravity mediated SUSY breaking. We find out that this possibility can be realized in the so-called focus-point region of parameter space. The mass degeneracy of higgsino-like chargino and two higgsino-like neutralinos is the necessary condition for a long lifetime. It requires the fine-tuning of parameters, but being a single additional constraint in the whole parameter space it can be fulfilled in the Constrained MSSM along the border line where radiative electroweak symmetry breaking fails. In a narrow band close to the border line the charginos are long-lived particles. The cross-sections of their production and co-production at the LHC via electroweak interaction reach a few tenth of $\mathrm{pb}$.
\end{abstract}

\section{Introduction}

An expected discovery of SUSY at the LHC is based on the MSSM predictions with a wide range of possibilities and uncertainties [1, 2, 3]. The usual pattern is that one has heavy short-lived particles which are created in strong and weak interactions and then decay (almost immediately) into Standard Model particles. These processes have a natural Standard Model background and their observation is rather sophisticated.

There are, however, some exotic cases, which are more natural within the gauge mediation scenario but may also be realized in mSUGRA when SUSY particles are long-lived. In the latter case, this usually takes place when their masses are degenerate with the LSP mass and the decay rate therefore is suppressed. This degeneracy in its turn requires precise fine-tuning of parameters of the mSUGRA parameter space. However, having at least four parameters $\left(m_{0}, m_{1 / 2}, A_{0}\right.$ and $\left.\tan \beta\right)$ one has enough freedom to satisfy this single additional requirement. 
In what follows we analyse the parameter space of the MSSM with gravity mediated SUSY breaking with the aim to find the regions where the above-mentioned degeneracy can take place and hence the long-lived superpartners might exist. In our previous papers we considered two of such possibilities: long-lived staus which live in the so-called coannihilation region [4, 5], and long-lived stops which live at the edge of the bulk region near the Higgs limit border line [6, 7].

Here we explore another region of parameter space which is a narrow band along the line where the radiative electroweak symmetry breaking fails. On the border of this region the Higgs mixing parameter $\mu$, which is determined from the requirement of electroweak symmetry breaking via radiative corrections, tends to zero. This leads to existence of light and degenerate states: the second chargino and two neutralinos, all of them being essentially higgsinos. Below we discuss their properties and possible creation at the LHC.

\section{Constraints on the MSSM Parameter Space for Large Values of $A$}

In what follows we consider the MSSM with gravity mediated supersymmetry breaking and the universal soft terms. We thus have the parameter space defined by $m_{0}, m_{1 / 2}, A, \tan \beta$ and we take the sign of $\mu$ to be positive motivated by contribution to the anomalous magnetic moment of muon [8, 9, 10]. Imposing the constraints like: i) the gauge couplings unification [11, 12, 13], ii) neutrality of the LSP [14, 15], iii) the Higgs boson and SUSY mass experimental limits [16, 17, 18], iv) radiative electroweak symmetry breaking [19, 20], we get the allowed region of parameter space of the CMSSM. Projected onto the $m_{0}, m_{1 / 2}$ plane this region depends on the values of $\tan \beta$ and $A$.

We are interested in chargino and neutralino masses. The mass terms are nondiagonal and look like

$$
\mathcal{L}_{\text {Gaugino-Higgsino }}=-\frac{1}{2} M_{3} \bar{\lambda}_{a} \lambda_{a}-\frac{1}{2} \bar{\chi} M^{(0)} \chi-\left(\bar{\psi} M^{(c)} \psi+\text { h.c. }\right),
$$

where $\lambda_{a}, a=1,2, \ldots, 8$, are the Majorana gluino fields and

$$
\chi=\left(\begin{array}{c}
\tilde{B}^{0} \\
\tilde{W}^{3} \\
\tilde{H}_{1}^{0} \\
\tilde{H}_{2}^{0}
\end{array}\right), \quad \psi=\left(\begin{array}{c}
\tilde{W}^{+} \\
\tilde{H}^{+}
\end{array}\right)
$$

are, respectively, the Majorana neutralino and Dirac chargino fields. 
At the tree level the neutralino mass matrix is

$$
M^{(0)}=\left(\begin{array}{cccc}
M_{1} & 0 & -M_{Z} \cos \beta \sin _{W} & M_{Z} \sin \beta \sin _{W} \\
0 & M_{2} & M_{Z} \cos \beta \cos _{W} & -M_{Z} \sin \beta \cos _{W} \\
-M_{Z} \cos \beta \sin _{W} & M_{Z} \cos \beta \cos _{W} & 0 & -\mu \\
M_{Z} \sin \beta \sin _{W} & -M_{Z} \sin \beta \cos _{W} & -\mu & 0
\end{array}\right),
$$

where $\tan \beta=v_{2} / v_{1}$ is the ratio of two Higgs v.e.v.s and $\sin _{W}=\sin \theta_{W}$ is the usual sinus of the weak mixing angle. The physical neutralino masses $m_{\tilde{\chi}_{i}^{0}}$ are obtained as eigenvalues of this matrix after diagonalization.

For charginos one has

$$
M^{(c)}=\left(\begin{array}{cc}
M_{2} & \sqrt{2} M_{W} \sin \beta \\
\sqrt{2} M_{W} \cos \beta & \mu
\end{array}\right) .
$$

This matrix has two chargino eigenstates $\tilde{\chi}_{1,2}^{ \pm}$with mass eigenvalues

$$
\begin{aligned}
M_{1,2}^{2} & =\frac{1}{2}\left[M_{2}^{2}+\mu^{2}+2 M_{W}^{2}\right. \\
& \left.\mp \sqrt{\left(M_{2}^{2}-\mu^{2}\right)^{2}+4 M_{W}^{4} \cos ^{2} 2 \beta+4 M_{W}^{2}\left(M_{2}^{2}+\mu^{2}+2 M_{2} \mu \sin 2 \beta\right)}\right] .
\end{aligned}
$$

These matrices obtain radiative corrections which are known in the leading order [21, 22]. Typically they are of the order of a few per cent.

When $\mu$ is small (less than $M_{Z}$ ), which takes place in the focus point region near the border line of radiative electroweak symmetry breaking, the lightest chargino $\left(\chi_{1}^{ \pm}\right)$and two lightest neutralinos $\left(\chi_{1,2}^{0}\right)$ are almost degenerate and have a mass of the order of $\mu$, which corresponds to the minus sign in Eq.5. All of them in this case are predominantly higgsinos. In Fig:1 it is shown how the mass of the lightest neutralino and the mass of the lightest chargino depend on $\mu$.

We are interested in the region of small $\mu$ where the three masses $\left(m_{\chi_{1}^{0}}, m_{\chi_{2}^{0}}, m_{\chi_{1}^{ \pm}}\right)$ are almost degenerate. This takes place for any choice of the other parameters since the tree level formulae weakly depend on them and the one-loop corrections are usually small. However, since the value of $\mu$ is not arbitrary in this approach but is taken from the requirement of electroweak symmetry breaking, one has to find the region of parameter space where it is small. In Fig:1 this region is just above the chargino LEP limit in the left bottom corner of the plots. One can see that masses are degenerate, and there the value of $\mu$ is of the order of $150-200 \mathrm{GeV}$ depending on the value of $\tan \beta$. There is also a slight dependence on $M_{2}$ (that is on $m_{1 / 2}$ ); however, this dependence only shows how far we may go along the lines having masses degenerate. It is clearly seen that the bigger $M_{2}$ the larger values of $\mu$ are allowed. The mass of $\chi_{2}^{0}$ is not shown, it almost coincides with the $\chi_{1}^{ \pm}$mass.

One has to check also that the other requirements on the values of the mSUGRA parameters are satisfied in this region. The well-known analysis of the CMSSM shows that small values of $\mu$ are achieved in the so-called focus-point region along 

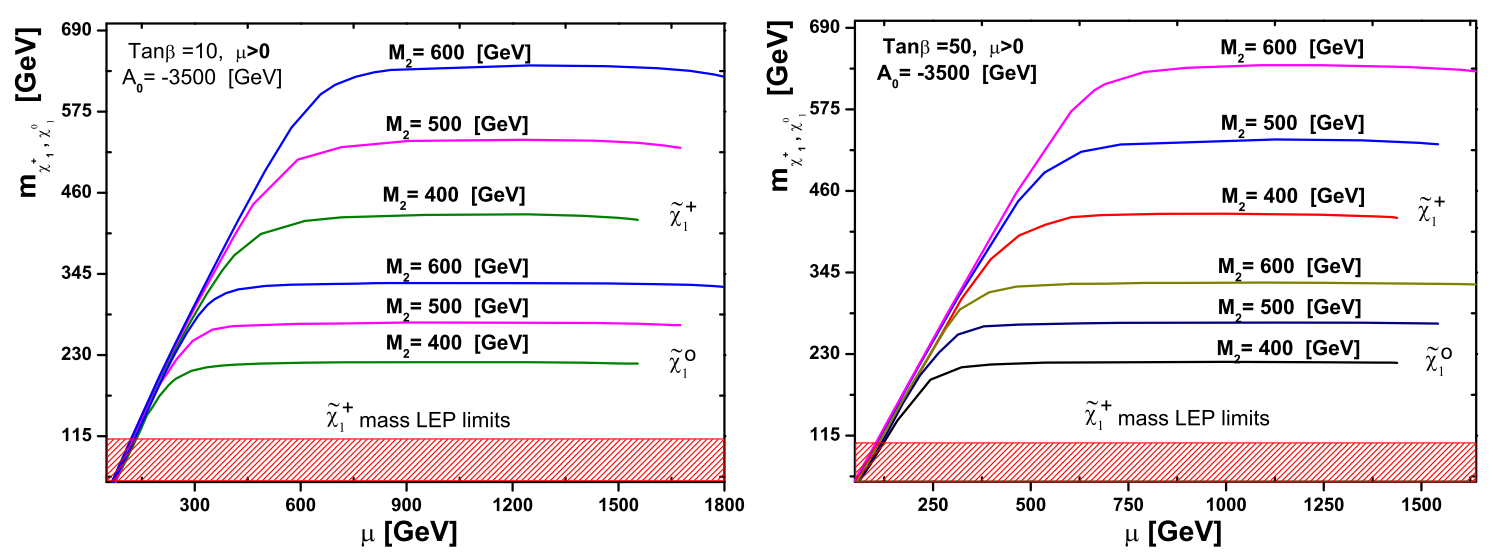

Figure 1: The masses of the lightest chargino and neutralino as functions of $\mu$ for the rest of parameters fixed. The value of $M_{2}$ is taken to be 600,500 and $400 \mathrm{GeV}$, and $\tan \beta=10,50$, respectively. Dark (red) lower band shows the experimental limit on chargino mass

the border line of electroweak symmetry breaking [23, 24, 25]. In Fig,2 we show the projection of SUSY parameter space onto the $m_{0}, m_{1 / 2}$ plane for different values of $A$ and $\tan \beta$. To calculate it, we use the SuSpect v2.3.4 code [26] 1 . The relic density was calculated with the help of the MicrOmegas package [27, 28]. In the case of light chargino and the second neutralino when they are almost degenerate with the lightest neutralino, when calculating the relic density one has to take into account not only the annihilation of two neutralinos but also the coannihilation of charginos and neutralinos.

One can see that for small values of $A_{0}$ the Dark matter line does not go along the electroweak symmetry breaking border but deviates from it, thus not allowing the small values of $\mu$. For large and negative $A_{0}$, on the contrary, these two lines almost coincide, the bigger the value of $\tan \beta$ the better.

It should be mentioned that the region near the border line is very sensitive to the Standard Model parameters; a minor shift in $\alpha_{s}$ or $m_{t}$ and $m_{b}$ leads to a noticeable change of the spectrum, as can be seen from comparison of different codes at [29, 30, 31].

Note that though the region of small $\mu$ looks very fine-tuned and indeed is very sensitive to all input parameters, still in the whole four dimensional parameter space (assuming universality) it swaps a wide area and can be easily reached. The accuracy of fine-tuning defines the accuracy of degeneracy of the masses and, hence, the life time of the NLSP which is the lighest chargino.

\footnotetext{
${ }^{1}$ We have set the following input values at $M_{W} ; \alpha_{e m}^{-1}=127.934, \alpha_{s}=0.1172, m_{t}^{\text {pole }}=172.7$ $\mathrm{GeV}, m_{b}\left(m_{b}\right)^{\overline{M S}}=4.25 \mathrm{GeV}, m_{\tau}^{\text {pole }}=1.777 \mathrm{GeV}$.
} 

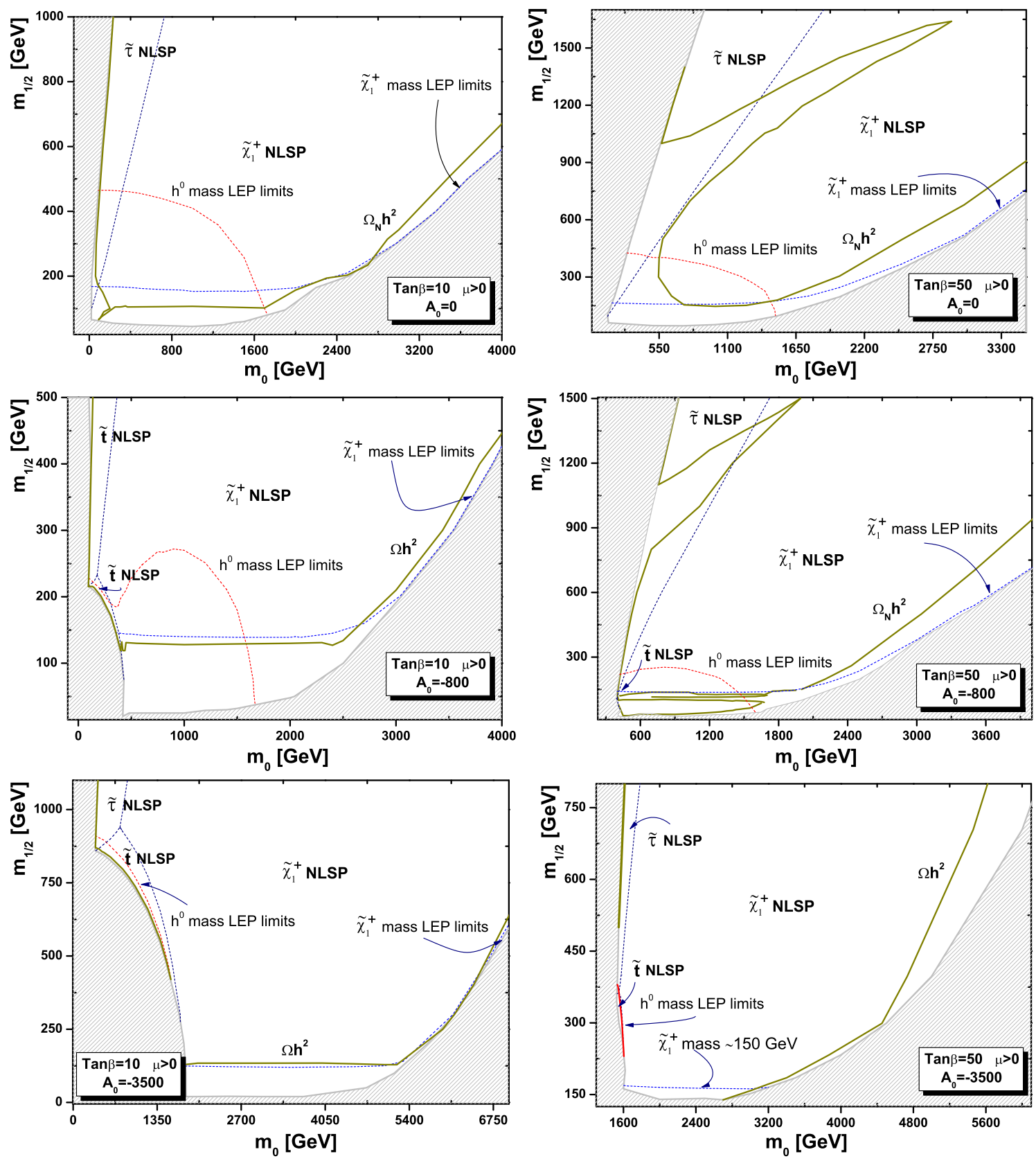

Figure 2: Allowed region of the mSUGRA parameter space for $A_{0}=0,-800,-3500$ $\mathrm{GeV}$ and $\tan \beta=10,50$, respectively. Dark (blue) areas show theoretically forbidden regions. Along the narrow green curve the amount of the Dark matter corresponds to WMAP data [32, 33] $\Omega h^{2}=0.09 \pm 0.04$. Experimental limits on the Higgs and chargino masses are shown as well. 


\section{Phenomenological Consequences of the Light Charginos}

Whence the parameters are chosen in such a way that one has mass degeneracy between the lightest chargino and the lightest neutralino and thus one has a longlived NLSP. Its mass is typically in the $100 \mathrm{GeV}$ range and the cross-section of production at the LHC is considerably high. The main production diagrams are shown in Fig 3. Since three states are almost degenerate, one has also co-production which has to be taken into account. This refers also to the annihilation process that defines the amount of the Dark matter.

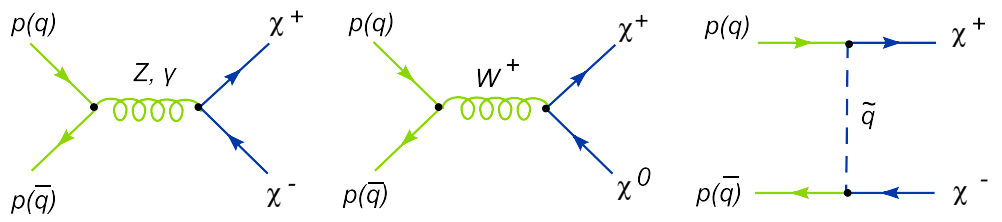

Figure 3: Main chargino production diagrams at the LHC

To calculate the production rate, one has to know the spectrum of the light states and the mixings in the chargino-neutralino sector. In our case, the lightest chargino and the lightest neutralinos are almost fully higgsinos. This property defines the preferences in the interaction pattern. To be specific, we choose several points in the mSUGRA parameter space which are situated in the focus point region along the border of electroweak symmetry breaking (see Fig 2 ). The values of $\mu$ and $\tan \beta$ are varied. As we have already mentioned for consistency with the relic density constraint the advocated scenario prefers large negative $A_{0}$ and large $\tan \beta$.

For each point we have calculated the cross-sections of chargino production and co-production with the lightest and next-to-lightest neutralino. The main reactions are

$$
p p \rightarrow \tilde{\chi}_{1}^{+} \tilde{\chi}_{1}^{-}, \quad p p \rightarrow \tilde{\chi}_{1}^{+} \tilde{\chi}_{1}^{0}, \quad p p \rightarrow \tilde{\chi}_{1}^{+} \tilde{\chi}_{2}^{0}
$$

The tree level processes are shown in Fig, 3 . The cross-sections are calculated with the help of the CALCHEP code [34] with the MRST parton distribution [35] and are summarized in Tables 1, 3. Shown also are the partial cross-sections for particular quarks inside the proton.

One can see that on average the cross-sections reach a few tenth of $\mathrm{pb}$ and slightly vary with the change of $\tan \beta$. The cross-sections mainly depend on $\mu$ : the bigger the value of $\mu$ the smaller the cross-section. This is due to the fact that the cross-section mostly depends on the masses determined mostly by $\mu$ and mixings.

The values of parameters used in our calculations are the following: we take $M_{1}=200 \mathrm{GeV}$, and all squark and slepton masses are around $1000 \mathrm{GeV}$. The values of $\mu$ are 120, 150 and $200 \mathrm{GeV}$, and $\tan \beta$ values are 10, 30, 50 . 


\begin{tabular}{|c|c|c|c|}
\hline Process & $\tan \beta=10$ & $\tan \beta=30$ & $\tan \beta=50$ \\
\hline \multirow{5}{*}{$\begin{array}{l}\bar{u} u \rightarrow \tilde{\chi}_{1}^{+} \tilde{\chi}_{1}^{-} \\
u \bar{u} \rightarrow \widetilde{\chi}_{1}^{+} \widetilde{\chi}_{1}^{-} \\
\bar{d} d \rightarrow \widetilde{\chi}_{1}^{+} \tilde{\chi}_{1}^{-} \\
d \bar{d} \rightarrow \widetilde{\chi}_{1}^{+} \widetilde{\chi}_{1}^{-}\end{array}$} & \multicolumn{3}{|c|}{$p p \rightarrow \widetilde{\chi}_{1}^{+} \tilde{\chi}_{1}^{-}$} \\
\hline & 0.46 & 0.45 & 0.45 \\
\hline & 0.46 & 0.45 & 0.45 \\
\hline & 0.20 & 0.19 & 0.19 \\
\hline & 0.20 & 0.19 & 0.19 \\
\hline$\sigma_{p_{1}}(p b)$ & 1.32 & 1.28 & 1.28 \\
\hline & \multicolumn{3}{|c|}{$p p \rightarrow \widetilde{\chi}_{1}^{+} \widetilde{\chi}_{1}^{0}$} \\
\hline$u \bar{d} \rightarrow \tilde{\chi}_{1}^{+} \tilde{\chi}_{1}^{0}$ & 0.82 & 0.79 & 0.78 \\
\hline $\bar{d} u \rightarrow \widetilde{\chi}_{1}^{+} \widetilde{\chi}_{1}^{0}$ & 0.82 & 0.79 & 0.78 \\
\hline$\sigma_{p_{2}}(p b)$ & 1.63 & 1.58 & 1.56 \\
\hline \multirow{3}{*}{$\begin{array}{l}u \bar{d} \rightarrow \tilde{\chi}_{1}^{+} \tilde{\chi}_{2}^{0} \\
\bar{d} u \rightarrow \widetilde{\chi}_{1}^{+} \widetilde{\chi}_{2}^{0}\end{array}$} & \multicolumn{3}{|c|}{$p p \rightarrow \widetilde{\chi}_{1}^{+} \widetilde{\chi}_{2}^{0}$} \\
\hline & 0.67 & 0.66 & 0.66 \\
\hline & 0.67 & 0.66 & 0.66 \\
\hline$\sigma_{p_{3}}(p b)$ & 1.34 & 1.32 & 1.32 \\
\hline
\end{tabular}

Table 1: The cross-sections for the chargino production and co-production at the $\mathrm{LHC}$ in the focus point region for $\mu=120 \mathrm{GeV}$ and $\tan \beta=10,30,50$

\begin{tabular}{|c|c|c|c|}
\hline Process & $\tan \beta=10$ & $\tan \beta=30$ & $\tan \beta=50$ \\
\hline \multirow{5}{*}{$\begin{array}{l}\bar{u} u \rightarrow \tilde{\chi}_{1}^{+} \tilde{\chi}_{1}^{-} \\
u \bar{u} \rightarrow \tilde{\chi}_{1}^{+} \tilde{\chi}_{1}^{-} \\
\bar{d} d \rightarrow \tilde{\chi}_{1}^{+} \tilde{\chi}_{1}^{-} \\
\bar{d} \bar{d} \rightarrow \widetilde{\chi}_{1}^{+} \tilde{\chi}_{1}^{-}\end{array}$} & \multicolumn{3}{|c|}{$p p \rightarrow \widetilde{\chi}_{1}^{+} \widetilde{\chi}_{1}^{-}$} \\
\hline & 0.22 & 0.22 & 0.21 \\
\hline & 0.22 & 0.22 & 0.21 \\
\hline & 0.09 & 0.09 & 0.09 \\
\hline & 0.09 & 0.09 & 0.09 \\
\hline$\sigma_{p_{1}}(p b)$ & 0.62 & 0.61 & 0.60 \\
\hline \multirow{3}{*}{$\begin{array}{l}u \bar{d} \rightarrow \tilde{\chi}_{1}^{+} \tilde{\chi}_{1}^{0} \\
\bar{d} u \rightarrow \widetilde{\chi}_{1}^{+} \widetilde{\chi}_{1}^{0}\end{array}$} & \multicolumn{3}{|c|}{$p p \rightarrow \tilde{\chi}_{1}^{+} \tilde{\chi}_{1}^{0}$} \\
\hline & 0.35 & 0.35 & 0.34 \\
\hline & 0.35 & 0.35 & 0.34 \\
\hline$\sigma_{p_{2}}(p b)$ & 0.70 & 0.69 & 0.68 \\
\hline \multirow{3}{*}{$\begin{array}{l}u \bar{d} \rightarrow \widetilde{\chi}_{1}^{+} \widetilde{\chi}_{2}^{0} \\
\bar{d} u \rightarrow \tilde{\chi}_{1}^{+} \widetilde{\chi}_{2}^{0}\end{array}$} & \multicolumn{3}{|c|}{$p p \rightarrow \widetilde{\chi}_{1}^{+} \widetilde{\chi}_{2}^{0}$} \\
\hline & 0.32 & 0.31 & 0.31 \\
\hline & 0.32 & 0.31 & 0.31 \\
\hline$\sigma_{p_{3}}(p b)$ & 0.64 & 0.63 & 0.62 \\
\hline
\end{tabular}

Table 2: The cross-sections for the chargino production and co-production at the $\mathrm{LHC}$ in the focus point region for $\mu=150 \mathrm{GeV}$ and $\tan \beta=10,30,50$ 


\begin{tabular}{|c|c|c|c|}
\hline Process & $\tan \beta=10$ & $\tan \beta=30$ & $\tan \beta=50$ \\
\hline \multirow{5}{*}{$\begin{array}{l}\bar{u} u \rightarrow \tilde{\chi}_{1}^{+} \tilde{\chi}_{1}^{-} \\
u \bar{u} \rightarrow \widetilde{\chi}_{1}^{+} \widetilde{\chi}_{1}^{-} \\
\bar{d} d \rightarrow \widetilde{\chi}_{1}^{+} \widetilde{\chi}_{1}^{-} \\
\overline{d \bar{d}} \rightarrow \widetilde{\chi}_{1}^{+} \widetilde{\chi}_{1}^{-}\end{array}$} & \multicolumn{3}{|c|}{$p p \rightarrow \widetilde{\chi}_{1}^{+} \widetilde{\chi}_{1}^{-}$} \\
\hline & 0.09 & 0.08 & 0.08 \\
\hline & 0.09 & 0.08 & 0.08 \\
\hline & 0.03 & 0.03 & 0.03 \\
\hline & 0.03 & 0.03 & 0.03 \\
\hline$\sigma_{p_{1}}(p b)$ & 0.23 & 0.22 & 0.22 \\
\hline \multirow{3}{*}{$\begin{aligned} u \bar{d} & \rightarrow \widetilde{\chi}_{1}^{+} \widetilde{\chi}_{1}^{0} \\
\bar{d} u & \rightarrow \widetilde{\chi}_{1}^{+} \widetilde{\chi}_{1}^{0}\end{aligned}$} & \multicolumn{3}{|c|}{$p p \rightarrow \widetilde{\chi}_{1}^{+} \widetilde{\chi}_{1}^{0}$} \\
\hline & 0.10 & 0.09 & 0.09 \\
\hline & 0.10 & 0.09 & 0.09 \\
\hline$\sigma_{p_{2}}(p b)$ & 0.20 & 0.19 & 0.19 \\
\hline \multirow{3}{*}{$\begin{array}{l}u \bar{d} \rightarrow \tilde{\chi}_{1}^{+} \tilde{\chi}_{2}^{0} \\
\bar{d} u \rightarrow \widetilde{\chi}_{1}^{+} \widetilde{\chi}_{2}^{0}\end{array}$} & \multicolumn{3}{|c|}{$p p \rightarrow \widetilde{\chi}_{1}^{+} \widetilde{\chi}_{2}^{0}$} \\
\hline & 0.13 & 0.12 & 0.12 \\
\hline & 0.12 & 0.12 & 0.12 \\
\hline$\sigma_{p_{3}}(p b)$ & 0.25 & 0.24 & 0.24 \\
\hline
\end{tabular}

Table 3: The cross-sections for the chargino production and co-production at the $\mathrm{LHC}$ in the focus point region for $\mu=200 \mathrm{GeV}$ and $\tan \beta=10,30,50$
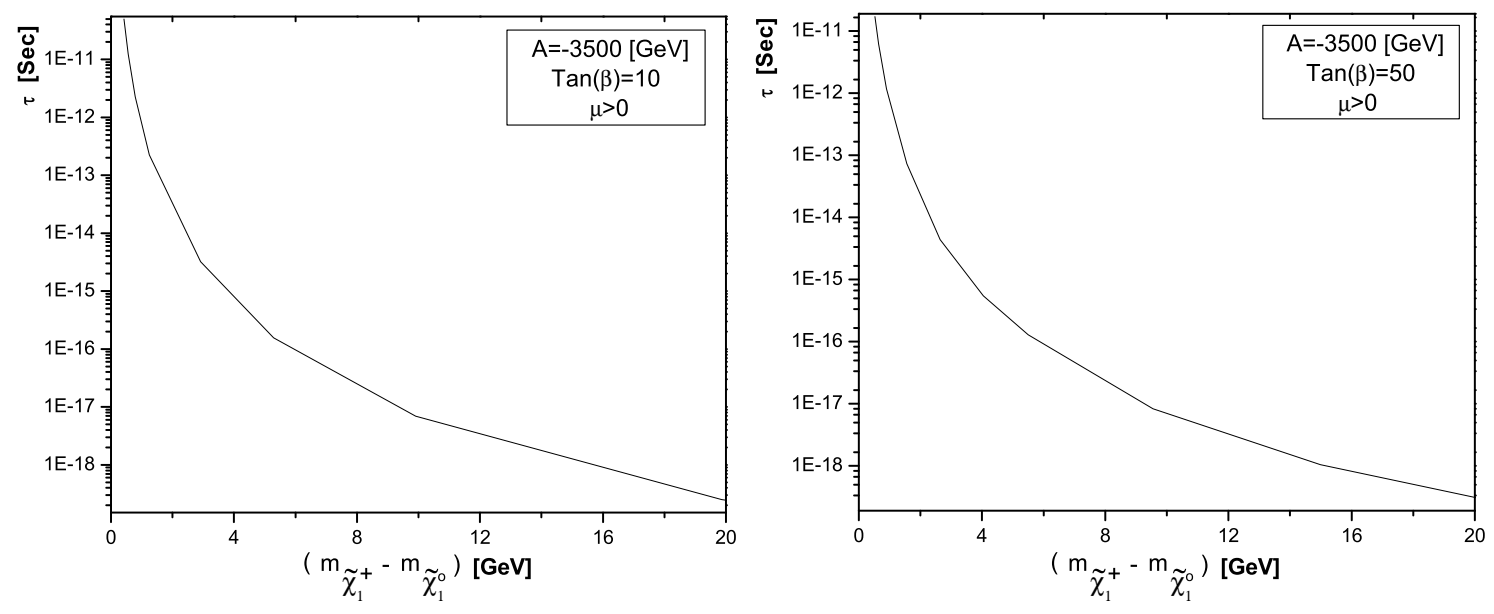

Figure 4: The lightest chargino lifetimes as a function of the mass difference between the lightest chargino (NLSP) and the lightest neutralino (LSP). 
In Fig. 4 we show the lifetime of the lightest chargino as a function of the mass difference between the lightest chargino (NLSP) and the lightest neutralino (LSP). It appears that in order to get reasonable "large" lifetimes one has to go very far along

the focus point region. Then keeping $\mu$ small one can get lifetimes of the order of $10^{-10} \mathrm{~s}$ for practically degenerate LSP and NLSP. When the mass difference increases the lifetime falls down. However, if the degeneracy is within a few $\mathrm{GeV}$, charginos are long-lived.

\section{Conclusion}

Experimentally, of course, the possibility to have a charged heavy long-lived particle is appealing. As well as in the case of long-lived stau and stop, which we considered in our previous papers [4, 5, 6, 7], we may conclude that within the framework of the MSSM with the soft supersymmetry breaking mechanism it is possible to get long-lived superpartners of the Higgs (or $W$-boson) which might be produced at the Large Hadronic Collider. The production cross-sections again crucially depend on a single parameter - the mass of the superparticle and for light charginos it can reach a few pb. As in the case of light stops, the light chargino NLSP scenario requires large negative values of the soft trilinear SUSY breaking parameter $A_{0}$, and large values of $m_{0}$ and $m_{1 / 2}$. The chargino decay would have an unusual signature and produce a noticable signal rather than pure missing energy taken away by the lightest neutralino. The two options are: charginos go through the detector, or they produce a secondary vertex when they decay inside the detector.

\section{Acknowledgements}

Financial support from RFBR grant \# 08-02-00856-a and grant of the Ministry of Education and Science of the Russian Federation \# 1027.2008.2 is acknowledged.

\section{References}

[1] H.P. Nilles, Phys. Rept. 110 (1984) 1.

[2] H.E. Haber, G.L. Kane, Phys. Rept. 117 (1985) 75.

[3] A.V. Gladyshev, D.I. Kazakov, Phys. Atom. Nucl. 70 (2007) 1553.

[4] A.V. Gladyshev, D.I. Kazakov, M.G. Paucar, Mod. Phys. Lett. A20 (2005) 3085.

[5] M.G. Paucar, Phys. Atom. Nucl. 71 (2008) 695.

[6] A.V. Gladyshev, D.I. Kazakov, M.G. Paucar, Light stops in the MSSM parameter space, arXiv:0704.1429v1 [hep-ph] 
[7] A.V. Gladyshev, D.I. Kazakov, M.G. Paucar, Long-living superpartners in the MSSM, in: Proceedings of the 15th International Conference on Supersymmetry and the Unification of Fundamental Interactions, eds. W. de Boer, I. Gebauer, p. 338, arXiv:0710.2322v1 [hep-ph]

[8] H.N. Brown et al. [Muon g-2 Collaboration], Phys. Rev. Lett 86 (2001) 2227.

[9] W. de Boer, M. Huber, C. Sander, D.I. Kazakov, Phys. Lett. B515 (2001) 283.

[10] W. de Boer, M. Huber, C. Sander, A.V. Gladyshev, D.I. Kazakov, A global fit to the anomalous magnetic moment, $b \rightarrow X_{s} \gamma$ and Higgs limits in the constrained MSSM, in Supersymmetry and unification of fundamental interactions: Proceedings, eds. D.I. Kazakov, A.V. Gladyshev (World Scientific, 2002), p. 196, hep-ph/0109131.

[11] U. Amaldi, W. de Boer, H. Furstenau, Phys. Lett. B260 (1991) 447.

[12] J.R. Ellis, S. Kelley, D.V. Nanopoulos, Phys. Lett. B260 (1991) 131.

[13] C. Giunti, C.W. Kim, U.W. Lee, Mod. Phys. Lett. A6 (1991) 1745.

[14] J.R. Ellis et al., Nucl. Phys. B238 (1984) 453.

[15] G. Jungman, M. Kamionkowski, K. Griest, Phys.Rep. 267 (1996) 195.

[16] LEP Higgs Working Group for Higgs boson searches, OPAL Collaboration, ALEPH Collaboration, DELPHI Collaboration and L3 Collaboration, Search for the Standard Model Higgs Boson at LEP, CERN-EP/2003-011, hep-ex/0306033.

[17] Joint LEP2 SUSY WG, Combined LEP Chargino Results up to $208 \mathrm{GeV}$, http://lepsusy.web.cern.ch/lepsusy/www/inos_moriond01/ charginos_pub.html

[18] Combined LEP Selectron/Smuon/Stau Results, 183-208 GeV, http://lepsusy.web.cern.ch/lepsusy/www/sleptons_summer02/ slep_2002.html

[19] L.E. Ibáñez, G.G. Ross, Nucl. Phys. B368 (1992) 3.

[20] G.G. Ross, R.G. Roberts, Nucl. Phys. B377 (1992) 571.

[21] D. Pierce, A. Papadopoulos, Phys. Rev. D50 (1994) 565.

[22] D.M. Pierce, J.A. Bagger, K.T. Matchev, R.J. Zhang, Nucl. Phys., B491 (1997) 3.

[23] J.L. Feng, K.T. Matchev, T. Moroi, Phys. Rev. Lett. 84 (2000) 2322. 
[24] J.L. Feng, K.T. Matchev, T. Moroi, Phys. Rev. D D61 (2000) 075005.

[25] H. Baer, C. Balazs, A. Belyaev, JHEP 3 (2002) 42.

[26] A. Djouadi, J.L. Kneur, G. Moultaka, Comp. Phys. Comm. 176 (2007) 426.

[27] G. Belanger, F. Boudjema, A. Pukhov, A. Semenov, MicrOMEGAs: Version 1.3, hep-ph/0405253.

[28] G. Belanger, F. Boudjema, A. Pukhov, A. Semenov, Comp. Phys. Comm. 149 (2002) 103.

[29] G. Belanger, S. Kraml, A. Pukhov, hep-ph/0502079.

[30] B.C. Allanach, S. Kraml, W. Porod, JHEP 03 (2003) 016.

[31] http://cern.ch/kraml/comparison/

[32] C.L. Bennett et al., Astrophys. J. Suppl. 148 (2003) 1.

[33] D.N. Spergel et al., Astrophys. J. Suppl. 148 (2003) 175.

[34] A. Pukhov, Calchep 3.2: MSSM, structure functions, event generation, and generation of matrix elements for other packages, hep-ph/0412191.

[35] A.D. Martin, R.G. Roberts, W.J. Stirling, R.S. Thorne, Eur. Phys. J. C28 (2003) 455. 